\title{
A refinement of Matrosov's theorem for differential inclusions
}

\author{
A.R. Teel ${ }^{\mathrm{a}}$,D. Nešić ${ }^{\mathrm{b}}$, T-C. Lee ${ }^{\mathrm{c}}$ and Y. Tan ${ }^{\mathrm{b}}$ \\ ${ }^{a}$ The Department of Electrical and Computer Engineering, Univ. of California, Santa Barbara, CA 93106, USA \\ ${ }^{\mathrm{b}}$ The Department of Electrical and Electronic Engineering, The University of Melbourne, Melbourne, VIC 3010, Australia \\ ${ }^{\mathrm{c}}$ The Department of Electrical Engineering, Minghsin University of Science and Technology, 1 Hsin-Hsing Road, Hsin-Fong, \\ Hsinchu, Taiwan 304, R.O.C
}

\begin{abstract}
This note presents a refinement of Matrosov's theorem for a class of differential inclusions whose set-valued map is defined as a closed convex hull of finitely many vector fields. This class of systems may arise in the analysis of switched nonlinear systems when stability with arbitrary switching between the given vector fields is considered. Assuming uniform global stability of a compact set, it is shown that uniform global attractivity of the set can be verified by tailoring Matrosov functions to individual vector fields. This refinement of Matrosov's theorem is an extension of the existing Matrosov results which may be easier to apply to certain differential inclusions than existing results, as demonstrated by an example.
\end{abstract}

\section{Introduction}

Matrosov's theorem allows one to check uniform asymptotic stability of time-varying systems in situations when uniform stability has already been established (Matrosov, 1963). In its original form (Matrosov, 1963), Matrosov's theorem establishes convergence via a positive definite Lyapunov function whose derivative is negative semi-definite and an auxiliary not necessarily sign-definite (Matrosov) function whose derivative is strictly negative on the neighbourhood of the set where the derivative of the Lyapunov function is equal to zero. A simpler version of Matrosov's theorem was presented in (Paden and Panja, 1988). Inspired by (Paden and Panja, 1988), a generalisation of this theorem with multiple auxiliary Matrosov functions was first proposed in (Loria et al., 2005). A Matrosov result for differential inclusions can be found in (Teel et al., 2002) but this result uses only two Matrosov functions. Results in (Sanfelice and Teel, 2009) apply to a class of differential inclusions but those results do not have the added flexibility of the current work, as it will be demonstrated via an example.

Email addresses: teel@ece.ucsb.edu (A.R. Teel), dnesic@unimelb.edu.au (D. Nešić), tc1120@ms19.hinet.net (T-C. Lee), yingt@unimelb.edu.au (Y. Tan).

1 This research was supported by NSF grant number ECCS1232035 and ECCS-1508757 and AFOSR grant numbers AFOSR FA9550-12-1-0127 and FA9550-15-1-0155.

${ }^{2}$ This research is supported by the Australian Research Council under the Discovery Grant DP120101144.

3 This work was supported by the NSC, Taiwan, R.O.C., under contract MOST 104-2221-E-159 -007.
As pointed out in (Sanfelice and Teel, 2009), Matrosov's theorem provides alternative conditions to invariance principles for asymptotic stability of time-invariant systems (Krasovskii, 1963; LaSalle, 1967).

Matrosov's theorem was extended to discrete-time systems (Teel et al., 2010), parameterized discrete-time systems (Nešić and Teel, 2004), hybrid systems (Sanfelice and Teel, 2009) and stochastic systems in (Teel, 2013b) and (Teel, 2013a). Various Lyapunov function constructions via Matrosov functions were presented in (Malisoff and Mazenc, 2007; Mazenc et al., 2009; Mazenc and Nešić, 2007) for continuous-time systems.

The main purpose of this paper is to present a refinement for Matrosov's theorem for a class of differential inclusions that may arise in the stability analysis of switched nonlinear systems. The set-valued map defining the considered class of inclusions is generated as a closed convex hull of a finite set of time-varying vector fields. We assume that a compact set $\mathcal{A}$ is uniformly globally stable for the inclusion and we prove uniform global attractivity of the set via the Matrosov functions. It turns out that constructing Matrosov functions directly for the inclusion is in general difficult. We show that if we construct functions that are Matrosov functions for each of the constituting vector fields, then this implies uniform global attractivity of the set $\mathcal{A}$; this can be much simpler than finding Matrosov functions directly for the inclusion as illustrated by an example. We illustrate our result by an example with two vector fields.

The paper is organised as follows. We first present Matrosov's Theorem for general differential inclusions that 
generalises results in (Loria et al., 2005) and it is essentially contained in (Sanfelice and Teel, 2009, Theorem 4.1). Compared to the results in (Teel et al., 2002) it allows for more than two functions and it uses a simplified condition from (Paden and Panja, 1988). A refinement of Matrosov's Theorem for a class of differential inclusions is given in Section 3; this is the main result of our paper. We illustrate our results by applying our main result to an example taken from (Lee et al., 2015). Summary is given in the last section.

\section{Matrosov theorem for differential inclusions}

In this section, we consider differential inclusions of the form:

$$
\dot{x} \in F(t, x),
$$

where $F: \mathbb{R}_{>0} \times \mathbb{R}^{n} \rightrightarrows \mathbb{R}^{n}$. A solution of a differential inclusion, defined by a set-valued mapping $F$ (see Equation (1)), is a locally absolutely continuous $R^{n}$-valued function defined on some interval of the form $[a, b)$, with $0 \leq a<b$ (where $b$ could be the infinity), such that the derivative of $x$ at the time instant $t$ is in the set $F(t, x(t))$ for almost all $t$ in $[a, b)$. The interval $[\mathrm{a}, \mathrm{b})$ is defined as $\operatorname{Dom}(x)$ and $a$ is denoted as $t_{0}(x)$. The solution $x$ is said to be complete if $b=\infty$, and it is said to be maximal if there is no other solution $y$ such that $\operatorname{Dom}(x)$ is contained in $\operatorname{Dom}(y)$ and $x(t)=y(t), \forall t \in \operatorname{Dom}(x)$.

For a set-valued function $F: \mathbb{R}_{>0} \times \mathbb{R}^{n} \rightarrow \mathbb{R}^{n}$, let $D(F):=\left\{x \in \mathbb{R}^{n} \mid F(t, x) \neq \emptyset, \forall t \geq 0\right\}$. The following assumption can be used to guarantee the existence of solutions of the system (1) from points $x \in D(F)$ (see (Filippov, 1988) for the existence of solutions and more detailed discussion in (Goebel et al., 2009)).

Assumption 1 A set-valued function $F: \mathbb{R}_{>0} \times \mathbb{R}^{n} \rightarrow$ $\mathbb{R}^{n}$ satisfy the following conditions:

(a) $D(F)$ is a nonempty open subset of $\mathbb{R}^{n}$.

(b) $F$ is outer semi-continuous ${ }^{4}$ on $\mathbb{R}_{\geq 0} \times D(F)$.

(c) For each $x \in D(F)$ and each $t \geq 0, F(t, x)$ is compact and convex.

(d) F is locally bounded on $\mathbb{R}_{\geq 0} \times D(F)^{5}$.

We show that an extension of the Matrosov's theorem for differential equation presented in (Loria et al., 2005) is easy to state for differential inclusions of the form (1), see Theorem 1. The set of solutions for this inclusion starting from an initial time $t_{0}$ with an initial condition $x_{0}=x\left(t_{0}\right)$ is denoted as $\mathcal{S}\left(t_{0}, x_{0}\right)$. Let $|\xi|$ denote the Euclidean norm of a vector $\xi \in \mathbb{R}^{n}$. Given a set $\mathcal{A} \subset \mathbb{R}^{n}$,

\footnotetext{
${ }^{4}$ For a set-valued function $F: \mathbb{R}_{\geq 0} \times \mathbb{R}^{n} \rightarrow \mathbb{R}^{n}$, it is said to be outer semicontinuous on $\mathbb{R}_{>0} \times D(F)$ if for any $(t, x) \in$ $\mathbb{R}_{\geq 0} \times D(F)$ and any sequence $\left\{\left(t_{n}, x_{n}\right)\right\}_{n \in \mathbb{N}} \subset \mathbb{R}_{\geq 0} \times D(F)$ with $\left(t_{n}, x_{n}\right) \rightarrow(t, x)$ as $n \rightarrow \infty$, and $y_{n} \in F\left(t_{n}, x_{n}\right) \rightarrow y$ as $n \rightarrow \infty$, then $y \in F(t, x)$.

${ }^{5}$ For a set-valued function $F: \mathbb{R}_{>0} \times \mathbb{R}^{n} \rightarrow \mathbb{R}^{n}$, it is said to be locally bounded on $\mathbb{R}_{>0} \times D(F)$ if for any $(t, x) \in$ $\mathbb{R}_{\geq 0} \times D(F)$, there exist a $\bar{\delta}(t, x)>0$ and a compact set $K(t, x) \subset \mathbb{R}^{n}$ such that $F(s, y) \subset K, \forall|s-t|<\delta,|y-x|<\delta$.
}

we use $|\xi|_{\mathcal{A}}:=\inf _{z \in \mathcal{A}}|\xi-z|$ to denote the distance of $\xi \in \mathbb{R}^{n}$ from $\mathcal{A}$.

Definition 1 We use the following stability notions for the differential inclusion (1):

- A compact set $\mathcal{A}$ is said to be uniformly stable (US) if for any $\epsilon>0$ there exists $\delta=\delta(\epsilon)$ such that we have that $\left|x_{0}\right|_{\mathcal{A}} \leq \delta$ implies that $|x(t)|_{\mathcal{A}} \leq \epsilon$ for all $\left(t_{0}, x_{0}\right) \in \mathbb{R}_{\geq 0} \times \overline{\mathbb{R}}^{n}, x \in \mathcal{S}\left(t_{0}, x_{0}\right)$ and $t \in \overline{\operatorname{Dom}}(x)$.

- $\mathcal{A}$ is said to be uniformly globally stable (UGS) if it is US and the solutions are uniformly globally bounded with respect to $\mathcal{A}$; that is, for any $\Delta>0$ there exists $M>0$ such that $\left|x_{0}\right|_{\mathcal{A}} \leq \Delta$ implies that $|x(t)|_{\mathcal{A}} \leq$ $M$ for all $\left(t_{0}, x_{0}\right) \in \mathbb{R}_{\geq 0} \times \mathbb{R}^{n}, x \in \mathcal{S}\left(t_{0}, x_{0}\right)$ and $t \in$ $\operatorname{Dom}(x)$.

- The set $\mathcal{A}$ is uniformly globally attractive (UGA) if for any strictly positive $\Delta, \epsilon$, there exists $T=T(\Delta, \epsilon)$ such that $\left|x_{0}\right|_{\mathcal{A}} \leq \Delta$ implies that $|x(t)|_{\mathcal{A}} \leq \epsilon$ for all $\left(t_{0}, x_{0}\right) \in \mathbb{R}_{\geq 0} \times \mathbb{R}^{n}, x \in \mathcal{S}\left(t_{0}, x_{0}\right)$ and $t \in \operatorname{Dom}(x)$ with $t \geq t_{0}+T$.

- The set $\mathcal{A}$ is uniformly globally asymptotically stable if it is $U G S$ and $U G A$.

Remark 1 It is worthwhile to highlight that in the definitions of stability properties, it is not assumed that solutions are complete, see a similar discussion in (Sanfelice and Teel, 2009).

To state Theorem 1, we need to introduce some notation. Let $F: \mathbb{R}_{>0} \times \mathbb{R}^{n} \rightrightarrows \mathbb{R}^{n}$, the strictly positive real numbers $(\delta, \Delta)$ and the compact set $\mathcal{A} \subset \mathbb{R}^{n}$ be given. Define $D=D(\delta, \Delta):=\left\{\xi \in \mathbb{R}^{n}:|\xi|_{\mathcal{A}} \in[\delta, \Delta]\right\}$. Let $\gamma>0$ and $\mathbb{B}^{n}:=\left\{\xi \in \mathbb{R}^{n}:|\xi| \leq 1\right\} ;$ then $\gamma \mathbb{B}^{n}=\left\{\xi \in \mathbb{R}^{n}:|\xi| \leq\right.$ $\gamma\}$.

Next, the Matrosov property and Matrosov function are defined.

Definition 2 (Matrosov property) A finite set of continuous functions $\left\{Y_{j}\right\}_{j=1}^{r}, Y_{j}: \gamma \mathbb{B}^{m} \times D \rightarrow \mathbb{R}$ for each $j \in\{1, \ldots, r\}$, is said to have the Matrosov property relative to $(\gamma, D)$ if, with the additional definitions $Y_{0} \equiv 0$ and $Y_{r+1} \equiv 1$, we have the following property:

$$
\text { For each } j \in\{0, \ldots, r\} \text {, if }(z, x) \in \gamma \mathbb{B}^{m} \times D \text { and }
$$$$
Y_{0}(z, x)=\ldots=Y_{j}(z, x)=0 \text { then } Y_{j+1}(z, x) \leq 0 \text {. }
$$

Remark 2 Due to $Y_{0} \equiv 0$, this property with $j=0$ implies that $Y_{1}(z, x) \leq 0$ for all $(z, x) \in \gamma \mathbb{B}^{m} \times D$; due to $Y_{r+1} \equiv 1$, the property with $j=r$ implies that there are no points $(z, x) \in \gamma \mathbb{B}^{m} \times D$ for which $Y_{1}(z, x)=\cdots=$ $Y_{r}(z, x)=0$.

Definition 3 A finite set of continuously differentiable functions $\left\{W_{j}\right\}_{j=1}^{r}$, where $W_{j}: \mathbb{R}_{\geq 0} \times \mathbb{R}^{n} \rightarrow \mathbb{R}$ for each $j \in\{1, \ldots, r\}$, are said to be Matrosov functions for $(F, D)$ if there exists a function $\phi: \mathbb{R}_{\geq 0} \times \mathbb{R}^{n} \rightarrow \mathbb{R}^{m}$, a positive real number $\gamma$, and continuous functions $Y_{j}$ : $\gamma \mathbb{R}^{m} \times D \rightarrow \mathbb{R}, j \in\{1, \ldots, r\}$ that have the Matrosov 
property relative to $(\gamma, D)$ and, for each $j \in\{1, \ldots, r\}$,

$$
\begin{gathered}
\max \left\{\left|W_{j}(t, x)\right|,|\phi(t, x)|\right\} \leq \gamma \\
\forall(t, x) \in \mathbb{R}_{\geq 0} \times D \\
\nabla_{t} W_{j}(t, x)+\left\langle\nabla_{x} W_{j}(t, x), \xi\right\rangle \leq Y_{j}(\phi(t, x), x) \\
\forall(t, x) \in \mathbb{R}_{\geq 0} \times D, \xi \in F(t, x) .
\end{gathered}
$$

Now we can state the main result of this section. This result is a direct generalisation of Theorem 1 in (Loria et al., 2005) and it is essentially contained in (Sanfelice and Teel, 2009, Theorem 4.1) and so its proof is omitted.

Theorem 1 For the differential inclusion (1), if the compact set $\mathcal{A}$ is $U G S$ and, for each pair of strictly positive real numbers $(\delta, \Delta)$, there exist Matrosov functions for $(F, D)$, then $\mathcal{A}$ is $U G A S$.

\section{A refinement of Matrosov's theorem for dif- ferential inclusions}

In this section, we consider differential inclusions (1) where the set-valued mapping $F$ can be written as

$$
F(t, x)=\operatorname{co}\left(\bigcup_{i=1}^{m_{f}} f_{i}(t, x)\right), \forall(t, x) \in \mathbb{R}_{\geq 0} \times \mathbb{R}^{n}
$$

for a given positive integer $m$ and functions $f_{i}: \mathbb{R}_{\geq 0} \times$ $\mathbb{R}^{n} \rightarrow \mathbb{R}^{n}, i \in\left\{1, \ldots, m_{f}\right\}$.

Remark 3 To guarantee Assumption 1, it only needs to assume that $f_{i}, i=1, \ldots, m_{f}$, are all continuous. Thus the existence of solutions of the system (3) can be ensured.

Such differential inclusions may arise in the analysis of switched systems where arbitrary switching between vector fields $f_{i}$ is considered. It turns out that constructing Matrosov functions satisfying the conditions of Theorem 1 is very hard even in the case when we have (3). The following result shows that for differential inclusions with $F$ as in (1), (3), UGAS can be checked by completing a simpler task in which we still want to find one set of Matrosov functions $W_{j}$ that works for every vector field $f_{i}$ but, for each vector field, we are allowed to find a different set of functions $Y_{i, j}$ that provide the bounds on the derivatives of $W_{j}$ and have the Matrosov property. Note that this does not necessarily imply the existence of one set of functions $Y_{j}$ that provide the required bounds on the derivatives of $W_{j}$ and have the Matrosov property as we show later in Remark 4. This refinement of Matrosov's theorem can considerably simplify the stability analysis of time-varying switched systems. It would be straightforward to extend this result to other classes of systems, such as discrete-time deterministic or stochastic systems; we do not report these results to keep the presentation short.

Before stating the main result, the following common Matrosov functions are defined.
Definition 4 Let $\left\{f_{i}\right\}_{1 \leq i \leq m_{f}}$ be a finite family of single valued functions from $\mathbb{R}_{>0} \times \mathbb{R}^{n} \rightarrow \mathbb{R}^{n}$. A finite set of continuously differentiable functions

$$
\left\{W_{j}: \mathbb{R}_{\geq 0} \times \mathbb{R}^{n} \rightarrow \mathbb{R}\right\}_{0 \leq j \leq r}
$$

is said to becommon Matrosov functions for all $\left(f_{i}, D\right), i=1, \ldots, m_{f}$ if there exist $m \in \mathbb{N}, \gamma>0$, $\gamma^{\prime}>0$ and a function $\phi: \mathbb{R}_{\geq 0} \times D \rightarrow \mathbb{R}^{m}$ such that for each $1 \leq i \leq m_{f}$, there exists a finite family of continuous functions: $\left\{Y_{i, j}: \gamma \mathbb{B}^{m} \times D \rightarrow \mathbb{R}\right\}_{0 \leq j \leq r}$ having the Matrosov property and the following conditions hold

$$
\begin{gathered}
|\phi(t, x)| \leq \gamma, \text { and }\left|W_{j}(t, x)\right| \leq \gamma^{\prime} \\
\forall(t, x) \in \mathbb{R}_{\geq 0} \times D, \\
\nabla_{t} W_{j}(t, x)+\left\langle\nabla_{x} W_{j}(t, x), f_{i}(t, x)\right\rangle \leq Y_{i, j}(\phi(t, x), x) \\
\forall(t, x) \in \mathbb{R}_{\geq 0} \times D, \forall 1 \leq j \leq r .
\end{gathered}
$$

In order to prove the main result, the following lemma is needed. For any sequence $\left\{Y_{j}: \gamma \mathbb{B}^{m} \times D \rightarrow \mathbb{R}\right\}_{j=1}^{r}$, we construct a new sequence with a reduced size: $\left\{Y_{j}^{r \rho}: \gamma \mathbb{B}^{m} \times D \rightarrow \mathbb{R}\right\}_{j=1}^{r-1}$ in a following way:

$$
\begin{aligned}
& Y_{j}^{r \rho}(z, x) \\
= & \begin{cases}Y_{j}(z, x) & \text { if } 1 \leq j \leq r-2, \forall r>2 \\
\rho Y_{r-1}(z, x)+Y_{r}(z, x) & \text { if } j=r-1, \forall r>1\end{cases}
\end{aligned}
$$

where $\rho>0$. Lemma 1 provides a basis of Induction using in the proof of main result (Theorem 2).

Lemma 1 Let $\left\{Y_{j}: \gamma \mathbb{B}^{m} \times D \rightarrow \mathbb{R}\right\}_{j=1}^{r}$ be continuous functions having the Matrosov property relative to $(\gamma, D)$. Then, the following statements hold:

(a) If $r=1$, there is a $\mu>0$ such that

$$
Y_{1}(z, x) \leq-\mu, \forall(z, x) \in \gamma \mathbb{B}^{m} \times D
$$

(b) If $r>1$, there is a $\rho_{0}>0$ such that for any $\rho \geq \rho_{0}$, $Y_{j}^{r \rho}(z, x)$ also has the same Matrosov property.

Proof. The proof of Statement (a) is completed by Contradiction. Assume that Statement (a) does not hold, there exists a sequence $\left\{z_{n}, x_{n}\right\}_{n \in \mathbb{N}} \subseteq \gamma \mathbb{B}^{m} \times D$ such that

$Y_{1}\left(z_{n}, x_{n}\right)>-\frac{1}{n}, \forall n \in \mathbb{N}$.

Since $\mathcal{A}, D$ and $\gamma \mathbb{B}^{m}$ are compact, there exists a subsequence: $\left\{z_{n_{k}}, x_{n_{k}}\right\}_{k \in \mathbb{N}}$ of $\left\{z_{n}, x_{n}\right\}_{n \in \mathbb{N}}$ that converges to a point $(\bar{z}, \bar{x}) \in \gamma \mathbb{B}^{m} \times D$. As $Y_{1}$ is a continuous function, it follows that

$0 \geq Y_{1}(\bar{z}, \bar{x})=\lim _{k \rightarrow \infty} Y_{1}\left(z_{n_{k}}, x_{n_{k}}\right) \geq \lim _{k \rightarrow \infty} \frac{-1}{n_{k}}=0$, 
as $Y_{1} \leq 0$ (see Remark 2). This indicates $Y_{1}(\bar{z}, \bar{x}) \equiv 0$. By the Matrosov property relative to $(\gamma, D)$ and $Y_{0} \equiv 0$, we have $Y_{2}(\bar{z}, \bar{x}) \leq 0$, which contradicts the fact that $Y_{2} \equiv 1$ due to $r=1$. This completes the proof.

Contradiction is used to prove Statement (b). Assume that Statement (b) does not hold. Then there exist two sequences $\left\{\rho_{n}: \rho_{n}>0\right\}_{n \in \mathbb{N}}\left(\rho_{n} \rightarrow \infty\right)$ and $\left\{z_{n}, x_{n}\right\}_{n \in \mathbb{N}} \subseteq \gamma \mathbb{R}^{m} \times D$ such that for each $n \in \mathbb{N}$,

$Y_{0}\left(z_{n}, x_{n}\right)=Y_{1}\left(z_{n}, x_{n}\right)=\ldots=Y_{r-2}\left(z_{n}, x_{n}\right)=0$,

and $Y_{r-1}^{r \rho_{n}}\left(z_{n}, x_{n}\right)=\rho_{n} Y_{r-1}\left(z_{n}, x_{n}\right)+Y_{r}\left(z_{n}, x_{n}\right)>0$, which indicates

$Y_{r-1}\left(z_{n}, x_{n}\right)>-\frac{Y_{r}\left(z_{n}, x_{n}\right)}{\rho_{n}}$.

Again, the compactness of $\mathcal{A}, D$ and $\gamma \mathbb{B}^{m}$ provides the existence of a subsequence $\left\{z_{n_{k}}, x_{n_{k}}\right\}_{k \in \mathbb{N}}$ of $\left\{z_{n}, x_{n}\right\}_{n \in \mathbb{N}}$ that will converge to a point $(\bar{z}, \bar{x}) \in \gamma \mathbb{B}^{m} \times D$. The continuity of $Y_{j}, 0 \leq j \leq r$, yields

$$
\begin{aligned}
Y_{0}(\bar{z}, \bar{x}) & =Y_{1}(\bar{z}, \bar{x})=\ldots=Y_{r-2}(\bar{z}, \bar{x})=0 \\
Y_{r-1}(\bar{z}, \bar{x}) & =\lim _{k \rightarrow \infty} Y_{r-1}\left(z_{n_{k}}, x_{n_{k}}\right) \\
& \geq-\lim _{k \rightarrow \infty} \frac{Y_{r}\left(z_{n_{k}}, x_{n_{k}}\right)}{\rho_{n_{k}}}=0 .
\end{aligned}
$$

Using the Matrosov property, we can show that $Y_{r-1}(\bar{z}, \bar{x})=0$ and $Y_{r}(\bar{z}, \bar{x}) \leq 0$. Hence two cases are considered.

If $Y_{r}(\bar{z}, \bar{x})=0$, it follows that $Y_{r+1}(\bar{z}, \bar{x}) \leq 0$. This contradicts the fact that $Y_{r+1} \equiv 1$.

If $Y_{r}(\bar{z}, \bar{x})<0$, by continuity of $Y_{r}$, it follows that

$Y_{r-1}^{r \rho_{n_{k}}}\left(z_{n_{k}}, x_{n_{k}}\right)=\rho_{n_{k}} Y_{r-1}\left(z_{n_{k}}, x_{n_{k}}\right)+Y_{r}\left(z_{n_{k}}, x_{n_{k}}\right)<0$

for all sufficiently large $k$, where from the Matrosov property, $Y_{r-1}\left(z_{n_{k}}, x_{n_{k}}\right) \leq 0$ was used. It contradicts the fact that $Y_{r-1}^{r \rho_{n_{k}}}\left(z_{n_{k}}, x_{n_{k}}\right)>0$. This completes the proof.

Theorem 2 (Common Matrosov functions) For the differential inclusion (1) where $F$ has the form in (3), if the compact set $\mathcal{A}$ is $U G S$ and for each pair of strictly positive real numbers $(\delta, \Delta)$ there exists a set of functions that are common Matrosov functions for all $\left(f_{i}, D\right)$, for all $i \in\left\{1, \ldots, m_{f}\right\}$, then $\mathcal{A}$ is $U G A S$.

Proof. Let $\Delta$ and $\epsilon$ be any positive constants. Since $\mathcal{A}$ is UGS, next step is to show that $\mathcal{A}$ is UGA. The proof is completed by contradiction. Assume that $\mathcal{A}$ is not UGA, for each $n \in \mathbb{N}$ (with $T=n$ (see Definition $1)$ ), there exist a sequence $\left\{t_{n}, x_{0 n}\right\}_{n \in \mathbb{N}}$ and a solution $x_{n} \in \mathcal{S}\left(t_{n}, x_{0 n}\right)$ such that

$$
\begin{aligned}
& \left|x_{0 n}\right|_{\mathcal{A}} \leq \Delta \\
\Rightarrow & \left\{\begin{array}{c}
\left|x_{n}(t)\right|_{\mathcal{A}}>\delta, \forall t \in\left[t_{n}, t_{n}+n\right] \subset \operatorname{Dom}\left(x_{n}\right) \\
\left|x_{n}(t)\right|_{\mathcal{A}} \leq M, \forall t \in \operatorname{Dom}\left(x_{n}\right)
\end{array},\right.
\end{aligned}
$$

where $\delta$ and $M$ come from Definition 1 . Let $D=$ $D(\delta, M)$. This indicates $x_{n}(t) \in D, \forall t \in\left[t_{n}, t_{n}+n\right]$.

Let $\left\{W_{j}\right\}_{1 \leq j \leq r}$ be common Matrsov functions for all $\left(f_{i}, D\right), i=1, \ldots, m_{f}$, and $\left\{Y_{i, j}\right\}_{1 \leq j \leq r}$ be the corresponding functions that have the Matrosov property relative to $(\gamma, D)$. By applying Lemma 1, it will show the contradiction.

When $r=1$, applying Lemma 1 as well as Definition 4 indicates that there exists $\mu>0$ such that

$$
\nabla_{t} W_{1}(t, x)+\nabla_{x} W_{1}(t, x) \xi \leq \sum_{i=1}^{m_{f}} \alpha_{i} Y_{i, 1}(\phi(t, x), x) \leq-\mu
$$

for any $(t, x) \in \mathbb{R}_{\geq 0} \times D$, where $\alpha_{i} \geq 0$ satisfying

$$
\sum_{i=1}^{m_{f}} \alpha_{i}=1
$$

and

$$
\xi=\sum_{i=1}^{m_{f}} \alpha_{i} f_{i}(t, x)
$$

Consequently,

$$
\begin{aligned}
\frac{d W_{1}\left(t, x_{n}(t)\right)}{d t} & =\nabla_{t} W_{1}\left(t, x_{n}(t)\right)+\nabla_{x} W_{1}\left(t, x_{n}(t)\right) \dot{x}_{n}(t) \\
& \leq-\mu
\end{aligned}
$$

for almost all $t \in\left[t_{n}, t_{n}+n\right]$. Integrating both sides of the above inequality over the interval $\left[t_{n}, t_{n}+n\right]$ obtains

$-2 \gamma^{\prime} \leq W_{1}\left(t_{n}+n, x_{n}\left(t_{n}+n\right)\right)-W_{1}\left(t_{n}, x\left(t_{n}\right)\right) \leq-n \mu$

for all $n \in \mathbb{N}$, where $\gamma^{\prime}$ comes from Definition 4. The contradiction is obtained when $n$ tends to infinity.

When $r \geq 2$, Statement (b) in Lemma 1 can be used to construct two new sequences: $\left\{W_{j}^{r \rho}\right\}_{j=1}^{r-1}$ and $\left\{Y_{i, j}^{r \rho}\right\}_{j=1}^{r-1}, \forall i=1, \ldots, m_{f}$, for all sufficiently large $\rho$ such that the conditions described in Definition 4 still hold with $r$ and $\gamma^{\prime}$ being replaced by $r-1$ and $\gamma^{\prime}(1+\rho)$, respectively. By continuing this process, we can reduce the number $r$ to $r=1$ and the same contradiction holds in this case. This completes the proof. 


\section{Example}

In this section, we revisit an example considered in (Lee et al., 2015) and apply to it the refined Matrosov's theorem (Theorem 2). Consider the time-varying differential inclusion with state $x \in \mathbb{R}^{2}$ given as

$$
\begin{aligned}
\dot{x} & \in \operatorname{co}\left(\bigcup_{i \in\{1,2\}} f_{i}(t, x)\right)=: F(t, x), \\
f_{i}(t, x) & :=-\left(e_{i} e_{i}^{T}+\alpha_{i}(t, x) J\right) x \quad i \in\{1,2\}, \\
e_{1} & :=\left[\begin{array}{l}
1 \\
0
\end{array}\right], \quad e_{2}:=\left[\begin{array}{l}
0 \\
1
\end{array}\right], \quad J:=\left[\begin{array}{cc}
0 & 1 \\
-1 & 0
\end{array}\right] .
\end{aligned}
$$

We assume the following conditions on the functions $\alpha_{i}$.

Assumption 2 For all $i \in\{1,2\}$, the functions $\alpha_{i}$ : $\mathbb{R}_{>0} \times \mathbb{R}^{2} \rightarrow \mathbb{R}$ are continuously differentiable and satisfy the following properties for each pair of strictly positive real numbers $(\delta, \Delta)$ :

(1) there exists $\nu>0$ such that $\forall t \geq 0$, the following inequality holds:

$$
|x| \in[\delta, \Delta]
$$

$\Longrightarrow \max \left\{\left|\alpha_{i}(t, x)\right|,\left|\nabla_{t} \alpha_{i}(t, x)\right|,\left|\nabla_{x} \alpha_{i}(t, x)\right|\right\} \leq \nu ;$

(2) there exist $T>0$ and $\epsilon>0$ such that for $i=1,2$ we have

$$
\left|e_{i}^{T} x\right| \in[\delta, \Delta] \Longrightarrow \int_{t}^{t+T} \alpha_{3-i}^{2}\left(s, e_{i} e_{i}^{T} x\right) d s \geq \epsilon, \forall t \geq 0 .
$$

The following result was established also in (Lee et al., 2015) using different techniques.

Corollary 1 Under Assumption 2, the origin of the differential inclusion (7) is UGAS.

Proof: We prove Corollary 1 by constructing Matrosov functions that satisfy conditions of Theorem 2. First, we show UGS. Consider a Lyapunov function candidate $V(x):=0.5 x^{T} x$. A simple calculation gives

$$
\left\langle\nabla V(x), f_{i}(t, x)\right\rangle=-x_{i}^{2} \leq 0 .
$$

Thus, for every $\lambda \in[0,1]$,

$$
\begin{aligned}
\langle\nabla V(x), \lambda & \left.f_{1}(t, x)+(1-\lambda) f_{2}(t, x)\right\rangle \\
& =-\lambda\left|x_{1}\right|^{2}-(1-\lambda)\left|x_{2}\right|^{2} \\
& \leq-\min \left\{\left|x_{1}\right|^{2},\left|x_{2}\right|^{2}\right\} \leq 0 .
\end{aligned}
$$

This bound and the fact that $V$ is independent of $t$, positive definite and radially unbounded implies that the origin is UGS.
It remains to establish that the origin is UGA. For this property, we use common Matrosov functions. We let $(\delta, \Delta)$ be a given pair of positive real numbers such that $0<\delta<\Delta$. Via Assumption 2, these numbers generate positive real numbers $\nu, T$, and $\varepsilon$. With the previous definition of $D$, for this example we have $D=$ $\left\{\xi \in \mathbb{R}^{2}:|\xi| \in[\delta, \Delta]\right\}$.

We start with the definitions

$$
\begin{aligned}
W_{1}(t, x) & :=V(x) \\
Y_{1,1}(z, x) & :=-x_{1}^{2} \\
Y_{2,1}(z, x) & :=-x_{2}^{2} .
\end{aligned}
$$

and observe that, due to (8), for $i \in\{1,2\}$,

$$
\begin{gathered}
\nabla_{t} W_{1}(t, x)+\left\langle\nabla_{x} W_{1}(t, x), f_{i}(t, x)\right\rangle \leq Y_{i, 1}(\phi(t, x), x) \\
\forall(t, x) \in \mathbb{R}_{\geq 0} \times D
\end{gathered}
$$

Let $W_{2}(t, x):=-x_{1} x_{2}\left(\alpha_{2}(t, x) x_{1}^{2}-\alpha_{1}(t, x) x_{2}^{2}\right)$. Then, direct computations show that there exists some $\beta_{2}>0$ that depends on $(\delta, \Delta)$ so that the following holds for $i=1,2$ and for all $(t, x) \in \mathbb{R}_{\geq 0} \times D$ :

$$
\begin{aligned}
& \nabla_{t} W_{2}(t, x)+\left\langle\nabla_{x} W_{2}(t, x), f_{i}(t, x)\right\rangle \\
\leq & -\alpha_{i}^{2}(t, x) x_{3-i}^{4}+\beta_{2}\left|x_{i}\right| .
\end{aligned}
$$

Let

$$
\begin{aligned}
W_{3}(t, x):= & -x_{2}^{2} \int_{t}^{\infty} \exp (t-\tau) \alpha_{1}^{2}\left(\tau, e_{2} e_{2}^{T} x\right) d \tau \\
& -x_{1}^{2} \int_{t}^{\infty} \exp (t-\tau) \alpha_{2}^{2}\left(\tau, e_{1} e_{1}^{T} x\right) d \tau
\end{aligned}
$$

Let $(0.5 \delta, \Delta)$ generate $\epsilon$ and $T$ from Assumption 2. Using our assumptions and following calculations as in (Loria et al., 2005), it can be shown that there exists $\beta_{3}>0$, which depends on $(\delta, \Delta)$ such that the following holds for $i=1,2$ and for all $(t, x) \in \mathbb{R}_{\geq 0} \times D$ :

$$
\begin{aligned}
& \nabla_{t} W_{3}(t, x)+\left\langle\nabla_{x} W_{3}(t, x), f_{i}(t, x)\right\rangle \\
\leq & -x_{2}^{2} \exp (-T) \int_{t}^{t+T} \alpha_{1}^{2}\left(\tau, e_{2} e_{2}^{T} x\right) d \tau \\
& -x_{1}^{2} \exp (-T) \int_{t}^{t+T} \alpha_{2}^{2}\left(\tau, e_{1} e_{1}^{T} x\right) d \tau \\
& +\beta_{3}\left(\left|x_{i}\right|+\left|\alpha_{i}(t, x) x_{3-i}\right|\right) \\
\leq & -x_{3-i}^{2} \exp (-T) \epsilon+\beta_{3}\left(\left|x_{i}\right|+\left|\alpha_{i}(t, x) x_{3-i}\right|\right)
\end{aligned}
$$

To show that the last inequality holds, we first consider $i=1$. This is done by considering two cases $\left|x_{2}\right|<\delta / 2$ and $\left|x_{2}\right| \geq \delta / 2$. Let $\left|x_{2}\right|<\delta / 2$. Then, since $|x|>\delta$, we have $\left|x_{1}\right| \geq \delta / 2$ and, hence, $-x_{1}^{2} \leq-x_{2}^{2}$ and

$$
\int_{t}^{t+T} \alpha_{2}^{2}\left(\tau, e_{1} e_{1}^{T} x\right) d \tau \geq \epsilon
$$


which implies

$$
-x_{1}^{2} \exp (-T) \int_{t}^{t+T} \alpha_{2}^{2}\left(\tau, e_{1} e_{1}^{T} x\right) d \tau \leq-x_{2}^{2} \exp (-T) \epsilon .
$$

When $\left|x_{2}\right| \geq \delta / 2$, then we have that

$$
\int_{t}^{t+T} \alpha_{1}^{2}\left(\tau, e_{2} e_{2}^{T} x\right) d \tau \geq \epsilon
$$

and hence

$$
-x_{2}^{2} \exp (-T) \int_{t}^{t+T} \alpha_{1}^{2}\left(\tau, e_{2} e_{2}^{T} x\right) d \tau \leq-x_{2}^{2} \exp (-T) \epsilon
$$

This completes the proof of the last inequality in (13) when $i=1$. The last inequality in (13) holds with $i=2$ because the equations are symmetric in index $i$. Hence, with the above defined $W_{j}(t, x), j=1,2,3$, we showed that we can use the following $Y_{i, j}: \gamma \mathbb{B}^{2} \times D \rightarrow \mathbb{R}, i=1,2$ and $j=1,2,3$ :

$Y_{i, 1}\left(z_{1}, z_{2}, x_{1}, x_{2}\right)=-x_{i}^{2}$

$Y_{i, 2}\left(z_{1}, z_{2}, x_{1}, x_{2}\right)=-z_{i}^{2} x_{3-i}^{4}+\beta_{2}\left|x_{i}\right|$

$Y_{i, 3}\left(z_{1}, z_{2}, x_{1}, x_{2}\right)=-x_{3-i}^{2} \exp (-T) \epsilon+\beta_{3}\left(\left|x_{i}\right|+\left|z_{i} x_{3-i}\right|\right)$

and it is easy to see that for each $i=1,2$ the function $\left(Y_{i, 1}, Y_{i, 2}, Y_{i, 3}\right)$ have the Matrosov property relative to $(\gamma, D)$, where

$\gamma:=\gamma^{\prime}=\sup _{t \geq 0, x \in D}\left[\sqrt{\alpha_{1}^{2}(t, x)+\alpha_{2}^{2}(t, x)}+V(x)+\bar{W}(t, x)\right]$

with

$\bar{W}(t, x):=\left|W_{2}(t, x)\right|+\left|W_{3}(t, x)\right|$.

From Theorem 2 it follows that the system is UGAS.

Remark 4 We note that if we define the functions

$$
Y_{j}(z, x):=\max _{i \in\left\{1, \ldots, m_{f}\right\}} Y_{i, j}(z, x),
$$

we have the condition (2b). However, these functions $Y_{j}$ do not have the Matrosov property relative to $(\gamma, D)$ for some $\gamma$ and $D$. For example, let $\delta>0$ and $\Delta>0$ be such that $\left(\frac{\gamma}{2},-\frac{\gamma}{2}, 0,1\right) \in \gamma \mathbb{B}^{2} \times D$. Now consider the point $\left(z_{1}, z_{2}, x_{1}, x_{2}\right)=\left(\frac{\gamma}{2},-\frac{\gamma}{2}, 0,1\right)$ and note that

$Y_{1}\left(\frac{\gamma}{2},-\frac{\gamma}{2}, 0,1\right)=0$

while

$$
Y_{2}\left(\frac{\gamma}{2},-\frac{\gamma}{2}, 0,1\right)=\beta_{2}
$$

where $\beta_{2}$ comes from (12). Therefore, in order to make use of the functions $\left(W_{1}, W_{2}, W_{3}\right)$, the refinement contained in Theorem 2 is clearly needed.

\section{Conclusions}

We provided a refinement of Matrosov's Theorem for differential inclusions that are generated using a convex hull of a finite number of vector fields. The usefulness of this result is illustrated by an example.

\section{References}

Filippov, A.F. (1988). Differential Equations with Discontinuous Righthand Sides. Kluwer.

Goebel, R., R. G. Sanfelice and A. Teel (2009). Hybrid dynamical systems. IEEE Control Systems Magazine 29, 28-93.

Krasovskii, N. N. (1963). Problems of the Theory of Stability of Motion. Translation of Russian ed. Moscow, Russia: Stanford Univ. Press, 1959.

LaSalle, J. P. (1967) An invariance principle in the theory of stability, in Differential Equations and Dynamical Systems New York: Academic, 277-286.

Lee, T.-C., Y. Tan and D. Nešić (2015). Stability and persistent excitation in signal sets. IEEE Trans. Autom. Control 60, 1188-1203.

Loria, A., E. Panteley, D. Popović and A. R. Teel (2005). A nested Matrosov theorem and persistency of excitation for uniform convergence in stable nonautonomous systems. IEEE Trans. Autom. Control 50, 183-198.

Malisoff, M. and F. Mazenc (2007). Constructions of strict Lyapunov functions for discrete time and hybrid time-varying systems. Nonlinear Anal.: Hybrid Syst. 2, 394-407.

Matrosov, V. M. (1963). On the stability of motion. J. Appl. Math. Mech 26, 1337-1353.

Mazenc, F. and D. Nešić (2007). Lyapunov functions for timevarying systems satisfying generalized conditions of Matrosov theorem. Math. Control Signals Syst. 19, 151-182.

Mazenc, F., M. Malisoff and O. Bernard (2009). A simplified design for strict Lyapunov functions under Matrosov conditions. IEEE Trans. Autom. Control, 54, 177-183.

Nešić, D. and A. R. Teel (2004). Matrosov theorem for parameterized families of discrete-time systems. Automatica 40, 1025-1034.

Paden, B. and R. Panja (1988). Globally asymptotically stable pd+controller for robot manipulators. Int. J. Control 47, 1692-1712.

Sanfelice, R. G. and A. R. Teel (2009). Asymptotic stability in hybrid systems via nested Matrosov functions. IEEE Trans. Autom. Control 54, 1569-1547.

Teel, A. R. (2013a). Lyapunov conditions certifying stability and recurrence for a class of stochastic hybrid systems. Annual Rev. Contr. 37, 1-24.

Teel, A. R. (2013b). A Matrosov theorem for adversarial Markov decision processes. IEEE Trans. Autom. Control 58, 21422148.

Teel, A. R., A. Loria and E. Panteley (2002). Integral characterizations of uniform asymptotic and exponential stability with application. Math. Contr., Sign. Syst., 15, 177201.

Teel, A. R., D. Nešić, A. Loria and E. Panteley (2010). Summability characterizations of uniform exponential and asymptotic stability of sets for difference inclusions. J. Diff. Equat. and Applic. 16, 173-194. 